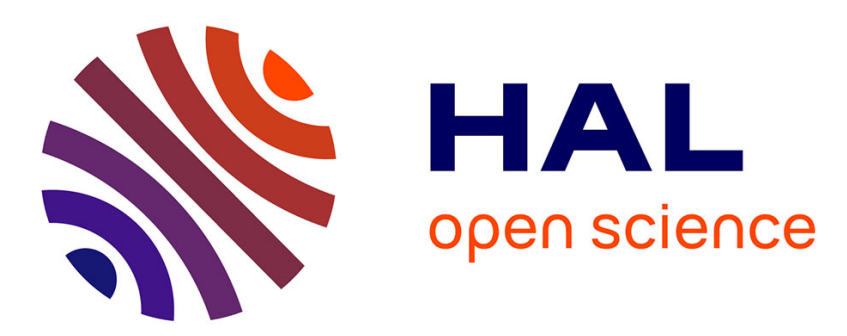

\title{
Modélisation de l'expansion d'une bulle dans le rotomoulage des mousses polymères par agent porogène chimique
}

\author{
Bassem Ben Lazreg, Francisco Chinesta, Abbas Tcharkhtchi
}

\section{To cite this version:}

Bassem Ben Lazreg, Francisco Chinesta, Abbas Tcharkhtchi. Modélisation de l'expansion d'une bulle dans le rotomoulage des mousses polymères par agent porogène chimique. Matériaux \& Techniques, 2008, 10.1051/mattech:2008048 . hal-01007069

\section{HAL Id: hal-01007069 \\ https://hal.science/hal-01007069}

Submitted on 22 Oct 2016

HAL is a multi-disciplinary open access archive for the deposit and dissemination of scientific research documents, whether they are published or not. The documents may come from teaching and research institutions in France or abroad, or from public or private research centers.
L'archive ouverte pluridisciplinaire HAL, est destinée au dépôt et à la diffusion de documents scientifiques de niveau recherche, publiés ou non, émanant des établissements d'enseignement et de recherche français ou étrangers, des laboratoires publics ou privés.

\section{(c)(1)}

Distributed under a Creative Commons Attribution| 4.0 International License 


\title{
Modélisation de l'expansion d'une bulle dans le rotomoulage des mousses polymères par agent porogène chimique
}

\author{
B. Ben Lazreg ${ }^{1,3}$, F. Chinesta ${ }^{2}$ et A. Tcharkhtchi ${ }^{3}$ \\ 1 Pôle Universitaire Léonard de Vinci-ESILV, 92916 Paris La Défense Cedex, France \\ e-mall : bassem.ben_lazreg@devinci.fr \\ 2 École centrale de Nantes, Laboratoire Matériaux, BP 92101, 44321 Nantes Cedex 3, France \\ e-mail : francisco chinesta@ec-nantes.fr \\ ${ }^{3}$ ENSAM - LIM, 151 Bd de l'Hôpital, 75013 Paris, France \\ e-mail : abbas.tcharkhtchi@paris.ensam.fr
}

\section{Mots-tiés :}

Rotomoulage; mousse polymère; agent porogène chimique; expansion de bulle; expansion libre de mousse

\section{Key words:}

Rotomolding; polymer foaming; chemical blowing agent; bubble growth; free expansion foam
Résumé - En rotomoulage, l'expansion des mousses est régie par la nucléation et l'évolution de la taille des bulles issues de la décomposition thermique des particules de l'agent moussant chimique. Les études expérimentales à l'échelle microscopique ont permis de tracer l'évolution cinétique d'une bulle au sein de la matrice fondue. Elles ont également démontré le caractère hétérogène des phénomènes de la nucléation, de l'expansion et de la distribution des bulles dans la matrice, La morphologie des mousses se trouve largement influencée par les parametres du procédé, les caractéristiques intrinsèques du polymère et ceux de l'agent porogène. L'évolution du rayon de la bulle est engendrée exclusivement par la décomposition de l'agent porogène solide situé à l'intérieur de la bulle. Cette décomposition se traduit par une augmentation du nombre de moles du gaz libérées, faisant ainsi croitre la pression du gaz au sein de la bulle. Un équilibre quasi-statique s'établit entre la pression du gaz, son volume, la pression extérieure, la viscosité et la tension de surface du polymère fondu. Les lois de conservation appliquées à cette cellule permettent de calculer l'évolution du rayon de la bulle au cours de la phase d'expansion ainsi qu'une comparaí son de notre modèle avec les résultats expérimentaux. Bien que ce modèle soit soumis à des hypothèses restrictives, il constitue une première étape vers l'étude d'un échantillon de mousse rotomoulée.

\begin{abstract}
Bubble expoansion modilling within polymer foam rotomolding by chemical blowing agent. During rotational foam moulding, the expansion of the polymer foam is governed by the nucleation and the growth of bubbles resulting from the thermal decomposition of solid chemical blowing agent particles. The experimental studies in microscopic scale made it possible to trace the kinetic evolution of a bubble within the molten matrix. These experiments showed also the heterogeneous character of the phenomena of nucleation, of the expansion of the bubbles and their distribution in the matrix. The morphology of the foam is largely influenced by the parameters of the process, the intrinsic characteristics of polymer and those of the blowing agent. The decomposition of the solid foaming agent results in an increase of the number of released gas moles. This increases the bubble gas pressure, a quasi-static balance is established between the pressure of gas, its volume, the external pressure, the viscosity and the surface tension of molten polymer. The conservation laws applied to a cell model make it possible to calculate the evolution of the bubble radius during expansion and a comparison of our model with the experimental results. Although this model is subjected to restrictive assumptions, it constitutes a first stage towards the study of rotomolded polymer foam sample.
\end{abstract}

es mousses thermoplastiques rotomoulées trouvent des applications industrielles de plus en plus variées compte tenu de leur rapport qualité prix et d'une large gamme de pièces que ce procédé peut réaliser. Le rotomoulage des mousses polymères est un procédé à basse pression qui utilise des agents porogènes chimiques pour la création de la phase gazeuse au sein de la matrice polymère fondue.

La taille et la distribution des alvéoles dépendent de la proportion de l'agent porogène, des conditions du procédé mais aussi des caractéristiques de la matrice et celles de l'agent porogène. La réaction de décomposition de l'agent porogène joue également un rôle important dans l'śolution de la phase gazeuse et, par conséquent, dans l'expansion de la mousse 
du fait qu'elle constitue l'élément moteur de l'opération de moussage dans ce procédé.

Le contrôle de ce processus de moussage nécessite une compréhension des interactions de ces différents paramètres. En effet, établir des modèles théoriques et des développements numériques en vue d'une simulation permet de réduire les coûts de production d'une part et de prédire le comportement mécanique des pièces moussées d'autre part.

Les travaux présentés ici s'inscrivent dans cette optique. Il s'agit d'étudier l'expansion de la mousse lors de sa mise en cuvre par un agent porogène chimique dans les conditions du rotomoulage à travers une approche microscopique.

Les études expérimentales microscopiques ont permis l'analyse des paramètres de la réaction de décomposition et du processus de l'expansion des bulles au sein d'une matrice visqueuse d'un thermoplastique à l'état fondu. Le matériau utilisé est le polyéthylène, compte tenu de son intérêt commercial. L'agent porogène chimique sélectionné est l'Azodicarbonamide, c'est un produit largement utilisé par les industriels du rotomoulage des mousses compte tenu de ses performances et de son prix.

La modélisation microscopique de l'expansion concerne, dans un premier temps, la cinétique chimique de la réaction de décomposition de l'agent porogène afin de déterminer l'évolution du nombre de moles de gaz. L'expansion proprement dite est étudiée à travers l'équilibre micromécanique d'une cellule élémentaire 2D formée d'une bulle unique. Par hypothèse, la bulle est considérée de forme sphérique et elle est entourée d'une matrice visqueuse à comportement new tonien.

\section{Cycle thermique de la mise en ceuvre de la mousse par rotomoulage}

Le moule, chargé de poudre est introduit dans le four maintenu à température constante. La chaleur est transférée progressivement par conduction depuis la surface externe du moule vers l'intérieur.

L'échauffement de l'air du moule contribue au passage du matériau de la température ambiante à sa température de fusion. La littérature a montré par expérience que la température à l'intérieur du four dépend des caractéristiques du moule et de l'épaisseur de la pièce.

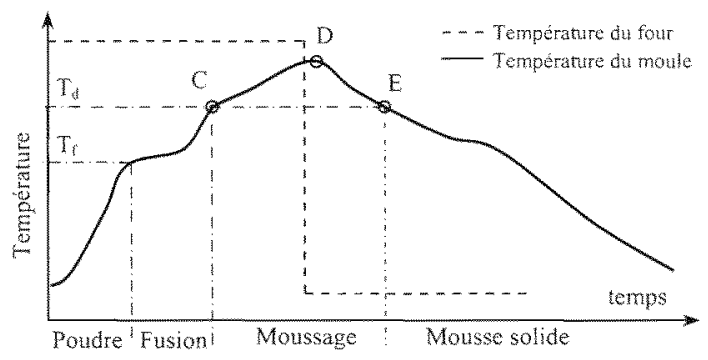

Fig. 1. Thermogramme expérimental typique d'un cycle de moussage (par le système Rotolog LIM- ENSAM Paris).

Fig. 1. Typical time-temperature profile in rotational foam molding cycle by rotolog system - LIM-ENSAM Paris).

Le suivi de l'évolution de la température de l'air à l'intérieur du moule au cours du procédé de moussage renseigne sur les changements d'état du polymère, en particulier, la fusion du polymère et la nucléation des bulles. Cela permet de distinguer les différents aspects macroscopiques du cycle thermique de la mise en cuvre des mousses.

La figure 1, ci-après, représente le profil expérimental typique de la température interne du moule tracé par le système "Rotolog " pendant le cycle du rotomoulage d'une mousse thermoplastique. Cette courbe permet l'identification des conditions thermiques du procédé [1].

A la température de fusion $T_{\mathrm{f}}$ du polymère, la première couche fondue adhère à la surface du moule. Au cours du chauffage, la température du polymère fondu augmente et sa viscosité diminue. Les particules de l'agent porogène aléatoirement dispersées dans la matrice sont encore à l'état solide.

A la température de décomposition de l'agent porogène, $T_{d}$, supérieure à la température de fusion du polymère, chacune des particules de l'agent porogène se dém compose, selon une réaction chimique, en une quantité de gaz qui se manifeste sous forme d'une bulle au sein de la matrice polymère fondue. La réaction de décomposition continue tant que la température à l'intérieur du moule est supérieure à $T_{d}$, et ce, jusqu'à l'épuisement total des particules de l'agent porogène. La quantité de gaz dégagée par la décomposition provoque l'expansion de la bulle.

Le point $C$ représente le début de la décomposition de lagent porogène et indique donc, le début du mécanisme de moussage. Celui-ci śarrête dès que la température décroît après le point $E$. En effet, la température devient inférieure à la température de 
décomposition de l'agent porogène, la décomposition de celui-ci s'arrête el la matrice commence à se cristalliser. Le point $\mathrm{D}$ représente le début de la phase de refroidissement. Il convient de noter que le refroidissement est relativement linéaire après une légère surélévation de la température provoquée par l'inertie thermique du système.

La mise en ceuvre de la mousse est donc déterminée par la partie (C, D et E) de la courbe qui est caractérisée par une variation linéaire de la température. Dans cet intervalle, la phase gazeuse apparaît et croît dans la matrice fondue [1].

Dans la pratique, ces rampes de température (chauffage et refroidissement) dépendent fortement des conditions du procédé telles que la température du four, le matériau du moule et son épaisseur, le mode de refroidissement, la présence d'inserts et de démoulant, la masse du matériau dans le moule, etc.

Une rampe de chauffage de l'ordre de $10^{\circ} \mathrm{C} / \mathrm{min}$ représente une valeur moyenne qui reflète les conditions thermiques du rotomoulage d'une pièce de dimensions moyennes $[1,2]$.

La réussite de l'opération de moussage par rotomoulage est conditionnée par une règle qui stipule que la température de décomposition $T_{d}$ del'agent porogène doit être supérieure à la température de fusion $T_{\mathfrak{f}} \mathrm{du}$ polymère, et ce, afin d'éviter une décomposition prématurée de l'agent porogène. La température maximale de la mise en ceuvre de la mousse (point D) doit être inférieure à la température de dégradation du polymère utilisé [3].

$$
\begin{aligned}
& T_{\text {coalescence des grains }}<T_{\text {fusion du polymère }} \\
& <T_{\text {décomposition de l'agent porogène }}<T_{\text {moussage }} \\
& <T_{\text {dégradation du polymère }}
\end{aligned}
$$

Bien que le moule soit animé d'une rotation continue sous une température supérieure à la température de fusion du polymère, la viscosité élevée du polymère fondu empêche son écoulement à l'intérieur du moule. 11 en résulte que la matrice n'est soumise à aucune sollicitation de cisaillement au cours du procédé du rotomoulage.

Par ailleurs, il convient de souligner que la courbe de température de la figure 1, ne fournit pas les variations thermiques locales du polymère mais plutôt, une valeur moyenne de la température de l'air prise à lintérieur du moule.

\section{Matériaux et méthodes}

Afin de comprendre l'interdépendance des différents paramètres du procédé de moussage, des expérimentations et des observations ont été effectuées aussi bien à l'échelle macroscopique qu'à l'échelle microscopique. Les divers matériaux et les méthodes utilisés ont permis de suivre les différentes étapes de la mise en cuvre d'une mousse polymère.

Lobjectif principal consiste à comprendre le mécanisme de moussage en fonction des différents paramètres du cycle du rotomoulage, notamment la cinétique de la décomposition de l'agent porogène.

Les travaux expérimentaux ont été réalisés essentiellement en utilisant l'Azodicarbonamide «Az120» qui est un agent porogène de la famille «Celogen » de la société Crompton/Uniroyal Chemicals.

C'est une substance organique qui se présente sous forme d'une poudre jaune dont la taille des particules est entre $2 \mu \mathrm{m}$ et $8 \mu \mathrm{m}$ avec une masse molaire de $116 \mathrm{~g}$. A la température $T_{d}$, qui est de $1^{\prime}$ ordre de $150^{\circ} \mathrm{C}$ à $160^{\circ} \mathrm{C}$, il se décompose en une quantité de gaz selon une réaction principale:

$$
\underbrace{\mathrm{C}_{2} \mathrm{H}_{4} \mathrm{~N}_{4} \mathrm{O}_{2}}_{1 \mathrm{Az} \text { (solide) }} \rightarrow \underbrace{\mathrm{N}_{2}+\mathrm{CO}+\mathrm{NH}_{3}}_{3(\mathrm{Gaz})}+\mathrm{HNCO}
$$

Cette réaction principale est suivie d'autres réactions secondaires de dimérisation et trimérisation de la molécule linéaire HNCO, qui donnent un résidu solide sous forme de poudre beige représentant environ $40 \%$ de la masse initiale. Cette masse résiduelle est souvent mentionnée dans la littérature $[2,3]$ comme un composé de cyamelide, d'acide cyanurique, d'Urazole et d'éventuels additifs et charges contenus dans l'Azodicarbonamide.

Dans la suite de notre étude, seule la réaction chimique principale (2) sera prise en compte, car elle est supposée suffisamment représentative de la décomposition de l'Azodicarbonamide dans les conditions de pression et de température du rotomoulage.

Le matériau moussant étudié est celui dénommé «M532» de la société "Matrix Polymers ". C'est un mélange de polyéthylène linéaire haute densité avec $1 \%$ d'Azodicarbonamide «Az120».

Les mesures effectuées sont essentiellement de trois types:

(i) des mesures enthalpiques différentielles par DSC Q1000 de la sociêté TA 


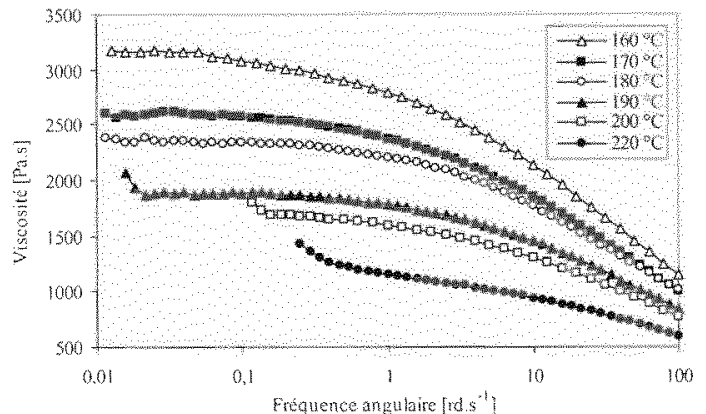

Fig, 2. Comportement du polymère fondu pour des differentes isothermes.

Fig. 2. Molten polymer behaviour for different isothermals.

et rhéologiques par Rhéomètre scientifique ARES à plateaux plats de la socété TA. Ces mesures concernent le polymère pur à l'état fondu. Compte tenu du fait que les analyses DSC du Polyéthylène sont bien connues, on limite l'étude à la présentation de quelques résultats des essais de rhéologie.

(ii) des mesures de perte de masse de l'agent porogène seul par thermogravimétrie «NETZSCH TG209 * afin d'évaluer la quantité de gaz libéré ainsi que la température de décomposition.

(iii) des mesures en 2D de l'expansion d'une bulle dans la matrice fondue à l'aide d'un microscope optique "Olympus BHZ-UMH » avec platine chauffante "METTLER FP * afin de tracer l'évolution du rayon en fonction de la température. Les observations ont été effectuées sur un grain du matériau moussant «M532» disposé entre deux lamelles et soumis à différentes rampes de température. L'acquisition et les traitements sont réalisés par le système « Microvision $\%$.

\section{Résultats et discussion}

\section{Caractéristiques théologiques de la matrice polymère}

Afin d'identifier le comportement de la matrice fondue, des essais rhéologiques ont été conduits sous différentes isothermes entre $160^{\circ} \mathrm{C}$ et $220^{\circ} \mathrm{C}$, ce qui correspond respectivement à la température du début et de la fin de décomposition de l'agent porogène. (Fig. 2).

On constate que le polyéthylène manifeste un caractère visqueux rhéofluidifiant.

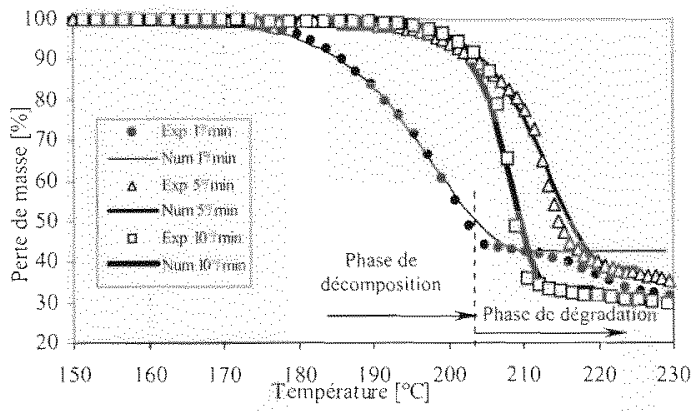

Fig. 3. Corrélation simulation/expérience de la perte de masse du « Az120».

Fig. 3. Correlation between numerical and experimental mass loss results of "Az120".

À faible taux de cisalllement, il présente un comportement newtonien. Les courbes de la figure 2 permettent de déterminer les paramètres de la thermodépendance de la viscosité.

\section{Décomposition de l'agent porogène}

\section{Essais de themogravimétrie}

L'Azodicarbonamide a été soumis a des essais de perte de masse sous des sollicitations thermiques isothermes et anisothermes. 11 s'agit de comprendre le mécanisme de décomposition afin d'identifier les caractéristiques de la réaction de décomposition dans les conditions du rotomoulage.

Lanalyse de la décomposition par la pesée de la masse confirme les résultats de la littérature [2]. Les fortes températures de chauffage augmentent la vitesse de décomposition del'agent porogene, et ce, aussibien en chauffage isotherme qu'en chauffage dynamique. Le démarrage du processus de décomposition est également influencé par la vitesse de chauffage.

Dans la figure 3, l'analyse des courbes expérimentales effectuées avec des vitesses de chauffage de $1^{\circ} \mathrm{C} / \mathrm{min}$ de $5^{\circ} \mathrm{C} / \mathrm{min}$ et de $10^{\circ} \mathrm{C} / \mathrm{min}$, met en évidence la perte de masse, conséquence de la décomposition de l'Azodicarbonamide selon la réaction principale (2). La phase de décomposition, relativement aux vitesses de $1^{\circ} \mathrm{C} / \mathrm{min}$, de $5^{\circ} \mathrm{C} / \mathrm{min}$ et de $10^{\circ} \mathrm{C} / \mathrm{min}$, se termine respectivement aux températures $204^{\circ} \mathrm{C}, 212^{\circ} \mathrm{C}$ et $218^{\circ} \mathrm{C}$.

A l'issue de cette réaction, on retrouve une masse résiduelle dont la valeur est entre $30 \%$ et $40 \%$ de la masse initiale.

Ces résultats corroborent ceux que nous trouvons dans la littérature. En poursuivant le chauffage, ces résidus se décomposent entièrement au voisinage de $300^{\circ} \mathrm{C}$. 
Les essais de perte de masse en isotherme fournissent les caractéristiques de la réaction chimique de la décomposition. En particulier, on détermine l'énergie d'activation $E_{k}$ et la constante de vitesse $k$ de la réaction principale de décomposition. Elles sont utiles dans la modélisation de la cinétique de la réaction.

\section{Modèle cinétique}

La réaction de décomposition (2) de l'agent porogène peut s'écrire sous la forme:

$$
1 \mathrm{Az} \rightarrow 3 \mathrm{G}+\mathrm{Q}
$$

G étant les moles de gaz dégagées de toute nature confondue.

L'équation cinétique de la réaction, que l'on considère d'ordre 1 , fournit la vitesse de la réaction $v(t)$ :

$$
v(t)=\frac{d[A z]}{d t}=-k(t)[A z]
$$

avec $[A z]$ la concentration de l'agent porogène, $t$ le temps et $k(t)$ la constante de vitesse de la réaction qui dépend de la température selon une loi d'Arrhénius:

$$
k(t)=k_{0} e^{\frac{k_{k} T(t)}{R_{2}}}
$$

$E_{k}$ étant l'énergie d'activation, $k_{0}$ est le facteur pré-exponentiel, $R_{g}$ est la constante des gaz parfaits.

En adoptant une croissance linéaire de la température $T(t)$ :

$$
\Gamma(t)=q t+T_{0}
$$

La perte de masse s'exprime alors par l'équation:

$$
\frac{d m}{m}=\frac{-k_{0}}{q} e^{\frac{k_{g} m_{m}}{k_{m}}} d T
$$

avec les conditions initiales suivantes:

- la température initiale $T(t=0)=T_{0}$.

- la masse initiale $m(t=0)=m_{0}$.

L'intégration de cette équation nécessite une démarche numérique qui, après validation par le cas isotherme, fournit le modèle cinétique de la décomposition de l'agent porogène dans les conditions du rotomoulage. La figure 3 montre la correspondance des résultats du modele proposé par rapport aux résultats expérimentaux de perte de masse. Ceux-ci s'obtiennent après un recalage des valeurs de l'énergie d'activation et de la constante de vitesse de la réaction de décomposition, en fonction de la rampe de température. Cette modélisation ne prend pas en compte la dégradation des résidus solides qui s'opère par d'autres mécanismes plus complexes.

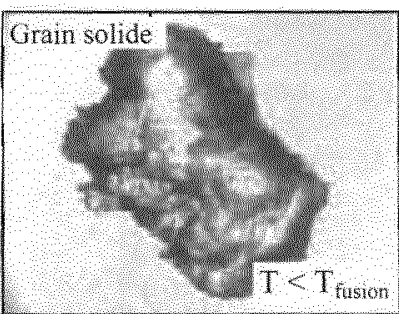

1. Etat initial du grain.

1. Initial pellet state.

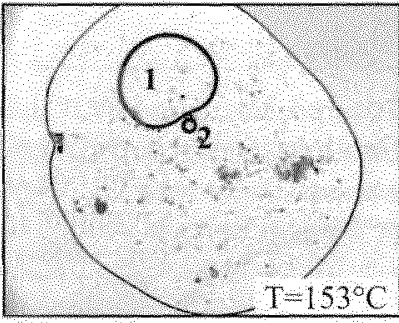

3. Nucléation de la bulle 2 . 3. Nucleation of bubble 2.

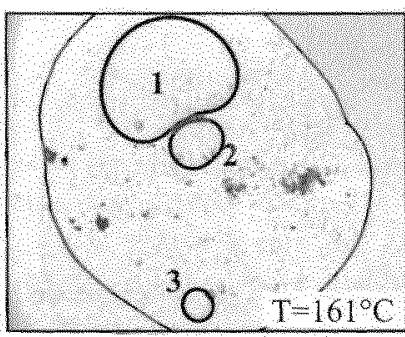

5. État stationnaire du rayon.

5. Stationary radius state.

Fig. 4 Observations $2 \mathrm{D}$ du moussage d'un grain M532. Fig. 4.2D Observation of a foaning M532 pellet.

\section{Expansion d'une bulle dans la matrice fondue}

\section{Observations sous microscope optique}

La figure 4 montre une microscopie $2 \mathrm{D}$ d'un grain moussant « M532* placé entre deux lamelles de verre et soumis à un chauffage avec une vitesse de $1^{\circ} \mathrm{C} / \mathrm{min}$. Ces photos montrent le mécanisme de moussage etillustrent les phénomènes de la nucléation et de l'expansion des bulles en fonction de la température dans une matrice de polymère fondue. La figure 4.1 montre la forme du grain dans son état solide dont la taille est de l'ordre de $0,5 \mathrm{~mm}$.

Après la fusion du polymère, on distingue sur la figure 4.2 la présence de taches noires qui représentent les particules de l'Azodicarbonamide aleatoirement dispersées dans la matrice. En effet la fusion d'un grain de polyéthylène (PE) pur (Fig. 5) fait 


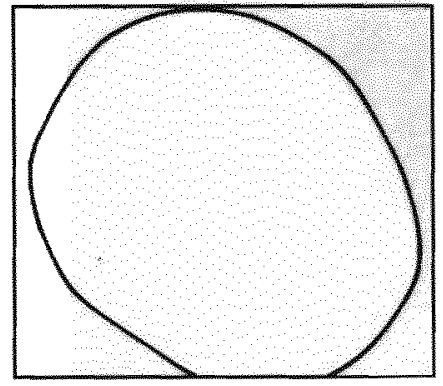

Fig. 5. Grain PE pur fondu.

Fig. 5. Pure PE molten pellet.

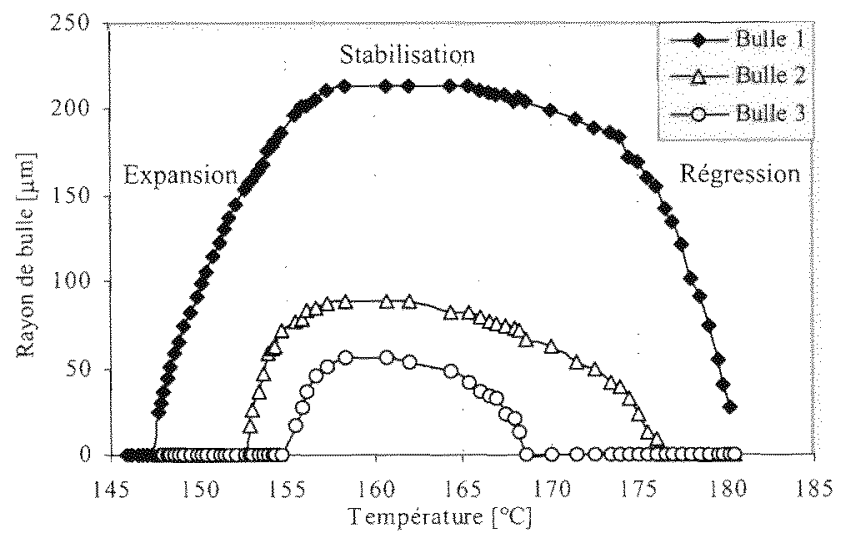

Fig. 6. Évolution des rayons de 3 bulles en fonction de la température $\left(1^{\circ} \mathrm{C} / \mathrm{min}\right)$.

Fig. 6. Evolution of three bubbles radiai as a function of temperature $\left(1^{\circ} \mathrm{C} / \mathrm{min}\right)$

apparaître un milieu homogène sans charges ni corps additionnels.

En fonction de la température on voit apparaitre la phase gazeuse d'une manière aléatoire à l'interface de certaines particules d'Azodicarbonamide. Les clichés 2 à 6 de la figure 4 montrent la nucléation de seulement 3 bulles par rapport à la quantité d'Azodicarbonamide disponible dans la matrice.

Ces observations mettent en évidence l'hétérogénéité du processus de moussage qui est dû essentiellement à l'hétérogénéité du champ de température et à une distribution aléatoire et non uniforme des particules de l'agent porogène dans la matrice polymère. Cette hétérogénéité est également liée aux tailles irrégulières des particules.

Un relevé des rayons des bulles 1,2 et 3 , nous a permis de tracer l'évolution du rayon en fonction de la température (Fig. 6).

La nature de l'évolution de ces courbes montre les étapes du cycle de vie d'une bulle quelconque dans les conditions du rotomoulage des mousses polymères. En effet, bien que l'évolution d'une bulle dépende de plusieurs paramètres, elle peut se résumer en quatre étapes principales:

\section{La nucléation}

L'amorçage de la réaction de décomposition fait apparaître une phase gazeuse qui se produit à l'interface des deux phases existantes [5]:

(i) la phase solide qui est la particule de l'agent porogène,

(ii) la phase liquide qui est le polymère fondu.

La densité de ces particules dans la matrice est directement liée au pourcentage de l'agent porogène utilisé. Chaque particule, selon sa taille et sa concentration, peut constituer un site de nucléation d'une bulle.

Si le volume de gaz engendré franchit le seuil critique défini par les caractéristiques physico-chimiques du mélange «matrice avec agent porogène $"$, une bulle sera alors engendrée.

\section{L'expansion}

Cette étape est définie par la croissance du volume de la bulle causée par l'augmentation du nombre de moles de gaz. Cette évolution est régie par la cinétique chimique de décomposition. L'expansion de la bulle continue progressivement tant que la température est supérieure à la température de décomposition de l'agent porogène, et ce, jusqu'à sa décomposition totale.

\section{La stabilisation}

C'est un état qui se caractérise par une taille stationnaire de la bulle. En effet, à la fin de la décomposition de l'agent porogène, le volume du gaz engendré atteint sa valeur maximale. Cette étape est considérée comme un état d'équilibre entre la pression du gaz au sein de la bulle d'une part et les contraintes de viscosité, de la tension de surface ainsi que la pression extérieure d'autre part.

La durée de cette étape peut être très courte voire inexistante pour certaines bulles. Elle dépend de la viscosité et des caractéristiques de diffusion de la matrice.

\section{La régression}

La décroissance du rayon de la bulle est provoquée par l'échappement des gaz à travers 
la matrice fondue. En effet, sous fortes températures, la viscosité et la tension de surface diminuent alors que les propriétés de perméabilité et de diffusion s'amplifient. À la fin de la décomposition de l'agent porogène, la diffusion des gaz à travers la matrice fondue devient alors prépondérante. Le gaz présent dans la bulle s'échappe et le rayon de celle-ci décroit jusqu'à sa disparition totale.

D'après les différentes observations microscopiques, on constate que :

- la nucléation et l'expansion des bulles se produisent dans un milieu fortement hétérogène. La comparaison entre un grain d'un mélange polyéthylène avec $1 \%$ d'Azodicarbonamide (Fig. 4.2) et celui de polyéthylène pur (Fig. 5), tous deux à l'état fondu, met en évidence le caractère aléatoire de la taille et de la distribution de ces charges,

- le champ de température est non uniforme, le gradient de température induit une forte hétérogénéité des phénomènes de nucléation et de l'expansion des bulles,

- l'expansion d'une bulle dépend du milieu environnant, de la température et de la concentration de l'agent porogène,

- la régularité de la forme géométrique des bulles et leur cycle de vie sont souvent altérés par la présence des charges, par la densité des bulles voisines et par la proximité des bords libres,

- on a rarement observé le phénomène de coalescence entre les bulles voisines, ceci s'explique par un état de contrainte qui reste inférieur à la charge de rupture du film de séparation des bulles.

\section{Modèle d'expansion d'une bulle}

La modélisation à l'echelle microscopique de l'expansion de la mousse, dans le contexte de notre procédé, commence par la modélisation de la cinétique chimique de la réaction de décomposition. Celle-ci constitue l'élément moteur de la génération de la phase gazeuse. Un modèle cellulaire [5] de la cinétique de la croissance d'une bulle dans une matrice polymère fondue (Fig. 7), constitue une première étape dans la simulation de l'opération de moussage par rotomoulage.

Vue la symétrie radiale du modèle cellulaire, on se place dans un repère sphérique, dont l'origine est au centre de la bulle (Fig. 8). Le domaine d'étude $\Omega$ est constitué de la fraction gazeuse $\Omega_{g}$ et la fraction polymère

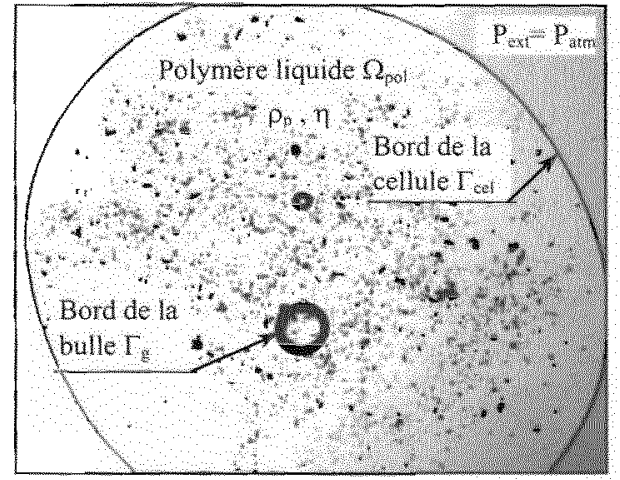

Fig. 7. Vue microscopique $2 \mathrm{D}$ d'un grain fondu: Description physique du modèle cellulaire.

Fig. 7. 2D microscopic view of molten grain: physical description of a unit foam cell model.

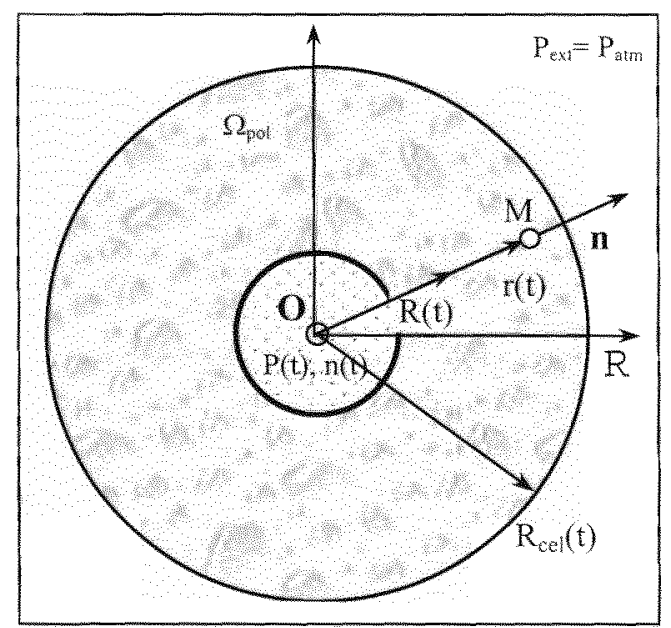

Fig. 8. Modèle de cellule élémentaire d'une mousse.

Fig. 8. Unit foam cell model.

$\Omega_{p o l}$ tels qu'à tout instant on a:

$$
\begin{gathered}
\Omega(t)=\Omega_{g}(t) \cup \Omega_{p o l}(t) \\
\Omega_{g}(t) \cap \Omega_{p o l}(t)=\Gamma_{g}(t) .
\end{gathered}
$$

L'étude de l'équilibre micromécanique de la matrice polymère suppose quelques hypothèses simplificatrices telles que:

(i) Le gaz et le polymère liquide sont non miscibles.

(ii) Le gaz est supposé comme un gaz parfait.

(iii) La cellule est considérée loin du contact du moule.

(iv) Les effets d'inertie sont nuls. La matrice est supposée un fluide visqueux incompressible newtonien.

(v) La nucléation, la diffusion et la dilatation des gaz ne sont pas prises en compte. 
(vi) La bulle, pendant sa croissance, a une forme sphérique de rayon $R(t)$. Elle est entourée d'un volume infini de polymère liquide pur dont les paramètres rhéologiques sont connus.

La matrice dans son écoulement, supposé du type laminaire, doit satisfaire les lois de conservation de la masse et de la quantité de mouvement établies en fonction des variables, le champ de vitesse $v(t)$ et le champ de pression $P(t)$.

Au bord de la bulle, la vitesse de la matrice est égale à la vitesse du rayon de la bulle :

$$
v(R, t)=\frac{d R(t)}{d t}=\dot{R}(t)
$$

L'expansion d'une bulle sphérique génère, dans la matrice fondue, un champ de vitesse purement radial $v(r, t)$ que l'on obtient par l'équation de continuité dans le système de coordonnées sphériques:

$$
v(r, t)=\frac{\dot{R}(t) R^{2}(t)}{r^{2}} .
$$

Cette cinématique est contrebalancée par les effets de la viscosité $\eta(T)$ de la tension de surface $\gamma$. L'état des contraintes dans la matrice s'exprime alors par la loi de comportement d'un fluide incompressible qui fait intervenir les contraintes de viscosité :

$$
\sigma_{i j}=-P(t) I+2 \eta(T) D_{i j}
$$

où $\eta(T)$ est le coefficient de viscosité de la matrice et $D_{i j}$ est le tenseur des vitesses de déformation.

Les conditions aux limites appliquées sont :

- au bord de la bulle $\Gamma_{g}=R$ :

$$
\sigma_{m}(R) n=-P(t) n+\frac{2 \gamma}{R} n
$$

- au bord de la cellule $T_{c e l}=R_{c e l}$ :

$$
\sigma_{r}\left(R_{c e l}\right) n=-P_{e x t} n
$$

où $n$ est le vecteur normal extérieur à la surface.

On obtient l'expression du rayon $R(t)$ de la bulle en fonction des paramètres théologiques de la matrice et de la pression du gaz $P(t)$ à l'intérieur de la bulle de la forme:

$$
P(t)-P_{e x t}=4 \eta(T) \frac{\dot{R}(t)}{R(t)}+\frac{2 \gamma}{R(t)} .
$$

En première approximation, on suppose que la tension de surface est constante et que la thermodépendance de la viscosité de la matrice suit la loi d'Arrhénius :

$$
\eta(T)=\eta_{0} e^{\frac{E_{\eta}}{R_{g}}\left(\frac{1}{T !}-\frac{1}{T_{0}}\right)}
$$

où 7o est la consistance, $E_{\eta}$ est l'énergie d'activation, $T_{0}$ une température de référence, et $R_{q}$ est la constante des gaz parfaits.

On voit, d'après l'équation (13), que la vitesse d'expansion d'une bulle est directement liée à la pression du gaz qui, par hypothèse, obeit à la loi des gaz parfaits.

L'analyse thermodynamique de la décomposition montre que l'évolution du fluide au cours du processus passe par une suite d'états d'équilibres gouvernés par les variables thermodynamiques telles que la pression, la température, le volume, l'énergie interne, etc.

A l'intérieur de la bulle, la pression est donnee, a tout instant, par l'équation d'état des gaz parfaits:

$$
P(t) V(t)=n(t) R_{g} T(t)
$$

où $n(t)$ est le nombre de moles de gaz. La réaction de décomposition (3) et les équations (4-6) forment le modèle qui exprime l'évolution du nombre de moles de gaz dans les conditions du rotomoulage.

On suppose qu'à l'instant initial $t=0$, la température est $T_{0}$, le rayon initial minimal nécessaire à la croissance de la bulle est $R_{0}$, la pression initiale est $P_{0}$ et le nombre de moles de gaz est noté $n_{0}$.

Au cours du processus de l'expansion, on suppose qu'à tout instant, on a :

$$
\frac{P(t) V(t)}{n(t) T(t)}=\frac{P_{0} V_{0}}{n_{0} T_{0}}=\text { Cst }
$$

Ce qui détermine l'expression de la pression en fonction du temps:

$$
P(t)=\frac{P_{0} V_{0}}{V(t)} \frac{n(t) T(t)}{n_{0} T_{0}}=\frac{P_{0} r_{0}^{3}}{r(t)^{3}} \frac{n(t) T(t)}{n_{0} T_{0}}
$$

Le modèle ainsi développé s'écrit par le système d'équations suivant :

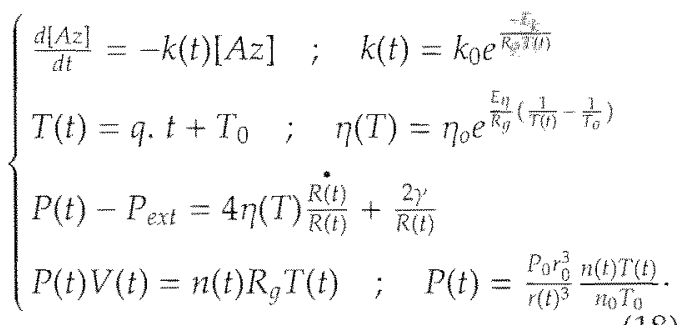

Sous les conditions d'un chauffage dynamique (Eq. (6)), ce modèle nécessite une 
Tableau 1. Valeurs des différents paramètres.

Table 1. Used parameters values.

\begin{tabular}{|c|c|c|c|}
\hline Nom & Symbole & Valeur & Unité \\
\hline $\begin{array}{l}\text { Constante de vitesse } \\
\text { de la réaction. }\end{array}$ & $k_{0}$ & $10^{24}$ & $s^{-1}$ \\
\hline Énergie d'activation & $E_{k}$ & 223800 & $\mathrm{Jmol}^{-1}$ \\
\hline Énergied'activation & $E_{11}$ & 27700 & $\mathrm{Jmol}^{-1}$ \\
\hline Tension de surface & $y$ & 0,03 & $\mathrm{Nm}^{-1}$ \\
\hline Viscosité à $200^{\circ} \mathrm{C}$ & 170 & 1200 & Pa.s \\
\hline Pression extérieure & $P_{a x t}=P_{a t n z}$ & $10^{5}$ & $\mathrm{~Pa}$ \\
\hline $\begin{array}{l}\text { Rayon initial } \\
\text { de la bulle }\end{array}$ & $R_{0}$ & $10^{-6}$ & $\mathrm{~m}$ \\
\hline
\end{tabular}

résolution numérique. La méthode utilisée est celle de Runge Kutta qui, après validation par la solution exacte du cas isotherme, foumit, sous des sollicitations thermiques non isothermes, l'évolution de la phase gazeuse et l'évolution du rayon d'une bulle. Les résultats présentés ci-dessous sont obtenus avec les valeurs des paramètres mentionnés dans le tableau 1.

\section{Cas isotherme}

Dans le cas isotherme, le système d'équations (18) admet une solution analytique. Une étude de sensibilité de cette solution aux différents paramètres permet de comprendre le processus de l'expansion de la bulle. Ci-dessous, sont présentées l'influence de la température et celle du rayon initial sur l'évolution du rayonet de la pression de la bulle. Les figures 9, 10 et 11 représentent l'effet de la température, respectivement sur le nombre de moles de gaz générées, sur le rayon et sur la pression de la bulle.

Cette étude montre que la température provoque deux effets majeurs :

(i) une augmentation de la vitesse de décomposition qui se traduit par un accroissement de la pression et une expansion plus rapide des bulles. Le tableau 2 présente l'évolution de la vitesse du nombre de moles $\frac{d n}{d t}$, celle de la vitesse du rayon $\frac{d R}{d t}$ et celle de la pression maximale, chacune obtenue pour les différentes courbes isothermes des figures 9,10 et 11

(ii) une diminution de la viscosité de la matrice qui contribue à une expansion plus facile de la bulle.

L'analyse de l'influence du rayon initial, en isotherme à $200^{\circ} \mathrm{C}$, montre que l'évolution du rayon de la bulle est très faiblement

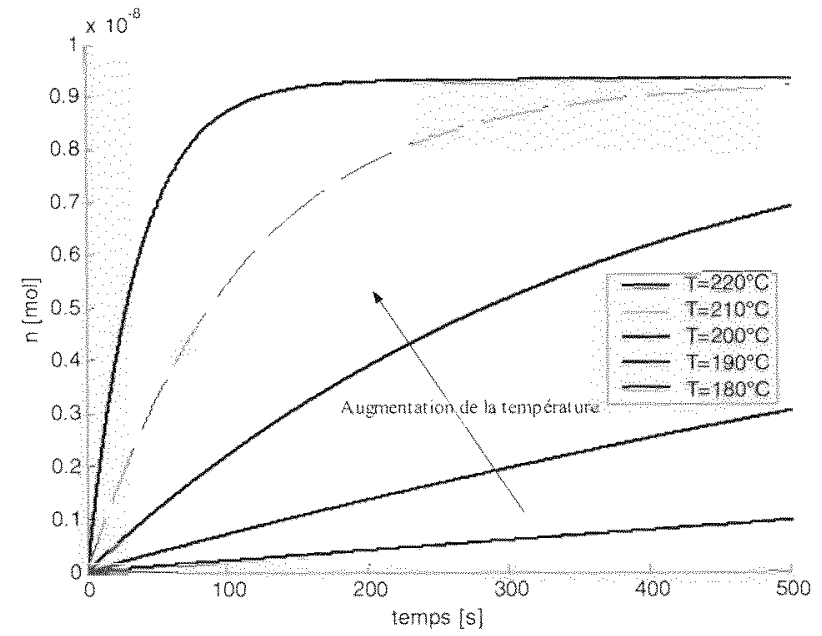

Fig. 9. Effet de la température sur le nombre de moles de gaz.

Fig. 9. Temperature effect on moles number of released gas.

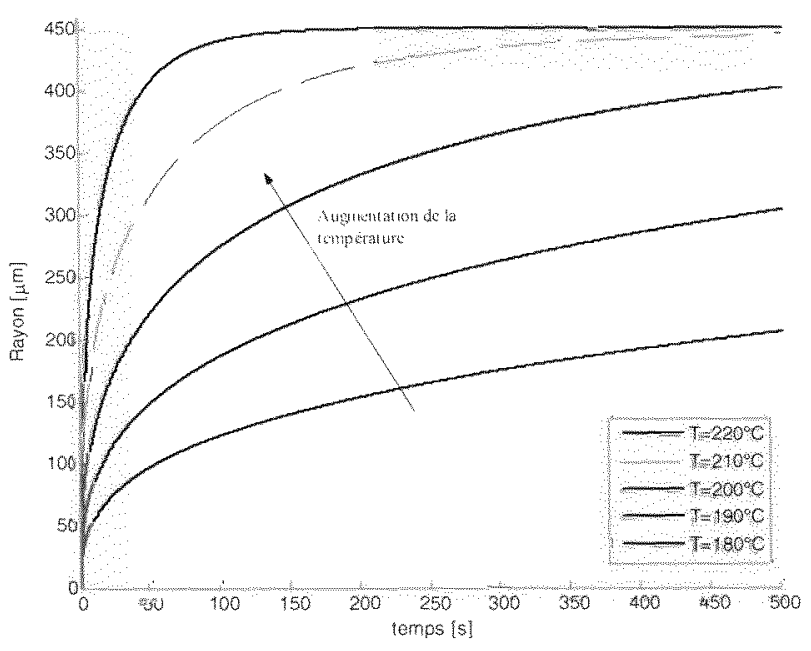

Fig. 10. Effet de la température sur le rayon d'une bulle. Fig. 10. Temperature effect on the bubble radius.

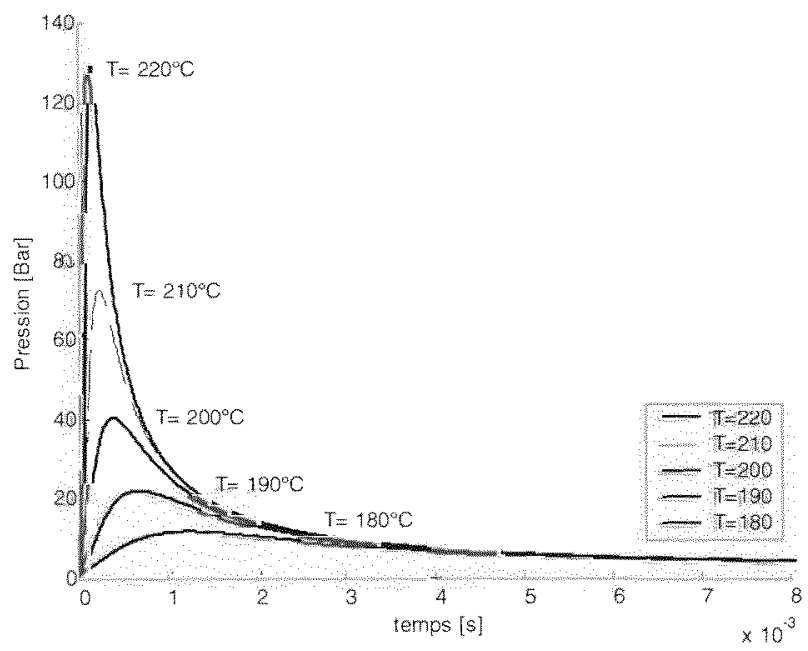

Fig. 11. Effet de la température sur la pression du gaz. Fig. 11. Temperature effect on the bubble pressure. 
Tableau 2. Influence de la température sur les paramètres d'expansion de bulle.

Table 2. Temperature effect on the bubble growth parameters.

\begin{tabular}{|c|c|c|c|}
\hline $\begin{array}{c}\mathrm{T} \\
{\left[{ }^{\circ} \mathrm{C}\right]}\end{array}$ & $\begin{array}{l}\text { La vitesse du } \\
\text { nombre de } \\
\text { moles } \\
{[\text { mol.s }} \\
\text {-1] }\end{array}$ & $\begin{array}{l}\text { La vitesse } \\
\text { d'expansion } \\
{\left[\mathrm{mm} \cdot \mathrm{s}^{-1}\right]}\end{array}$ & $\begin{array}{c}\text { Pression } \\
\text { maximale } \\
\text { [bar] }\end{array}$ \\
\hline 180 & $2 \times 10^{-12}$ & 0,3 & 12 \\
\hline 190 & $7 \times 10^{-12}$ & 0,7 & 22 \\
\hline 200 & $23 \times 10^{-12}$ & 1,1 & 40 \\
\hline 210 & $70 \times 10^{-12}$ & 1,8 & 73 \\
\hline 220 & $200 \times 10^{-12}$ & 3,7 & 129 \\
\hline
\end{tabular}

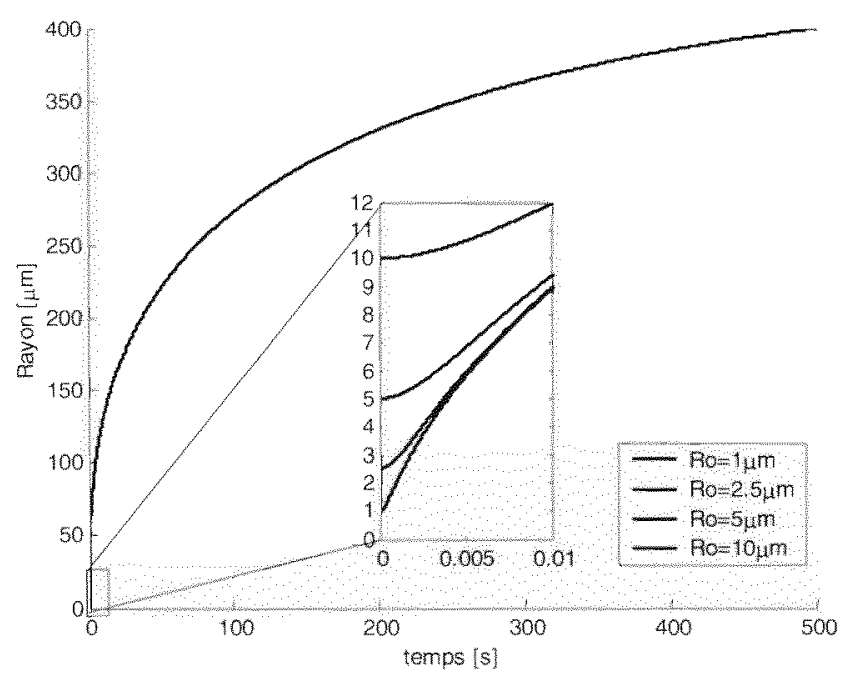

Fig. 12. Effet du rayon initial $R_{0}$ sur l'évolution du rayon de la bulle.

Fig. 12. Effect of the initial bubble radius $R_{0}$ on the bubble radius growth.

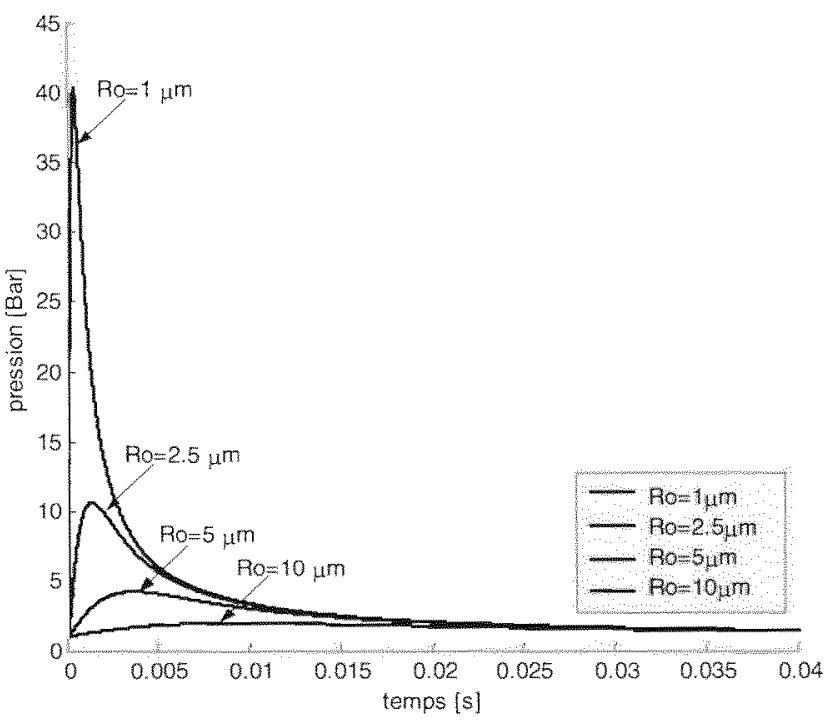

Fig. 13. Effet du rayon initial $R_{0}$ sur l'évolution de la pression.

Fig. 13. Initial bubble radius $R_{0}$ effect on the bubble pressure.
Tableau 3. Influence du rayon initial sur la pression.

Table 3. Initial bubble radius effect on the bubble pressure.

\begin{tabular}{|c|c|}
\hline$R_{0}$ [micron] & Pression maximale [bar] \\
\hline 1 & 40 \\
\hline 2,5 & 11 \\
\hline 5 & 4,25 \\
\hline 10 & 2 \\
\hline
\end{tabular}

affectée par la valeur du rayon initial. La figure 12 représente la courbe $R(t)$ pour les valeurs de $R_{0}$, respectivement $1 \mu \mathrm{m}, 2,5 \mu \mathrm{m}$, $5 \mu \mathrm{m}$ et $10 \mu \mathrm{m}$. Les quatre courbes sont superposées bien que le zoom mette en évidence les différentes valeurs du rayon initial. En revanche, une augmentation du rayon initial détend le gaz et fait décroître la pression (Fig. 13). On enregistre une pression maximale de 40 bars pour un rayon initial de $1 \mu \mathrm{m}$, alors qu'elle $n^{\prime}$ excède pas 2 bars avec un rayon initial de $10 \mu \mathrm{m}$. Le tableau 3 montre l'effet du rayon initial sur la pression du gaz.

Une comparaison entre la solution exacte et la solution numérique dans les cas isothermes permet d'étudier le comportement du schéma numérique adopté. On présente sur la figure 14, la correspondance entre la solution numérique (où la rampe de chauffage est nulle) et la solution analytique, dans le cas de $l^{\prime}$ isotherme $200^{\circ} \mathrm{C}$. L'erreur relative constatée est inférieure à $10^{-4}$.

\section{Cas anisotherme}

On constate que la décomposition et le rayon de la bulle dépendent fortement de la rampe de température. À la fin de la décomposition de l'agent porogène, le nombre de moles générées se maintient constant (Fig. 15), ce qui explique le changement du régime de la courbe $R(t)$ de la figure 16. En l'absence de la diffusion dans la simulation, le volume du gaz continue à croître à cause de l'augmentation de la température (Fig. 16).

Les résultats présentés ci-dessous ont été réalisés pour une température initiale $T_{0}=$ $150{ }^{\circ} \mathrm{C}$, un rayon initial $R_{0}=1 \mu \mathrm{m}$ et une pression initiale $P_{0}=P_{\text {atm }}$. 


\section{Vérification du modèle}

La confrontation entre la courbe numérique et la courbe expérimentale du rayon d'une bulle met en évidence une bonne corrélation entre le modèle et les mesures effectuées en 2D. La figure 17 montre le cas de la bulle 1 présentée précédemment sur la figure 6 .

À partir de cette superposition, les commentaires suivants découlent :

(1) Cette modélisation ne prend pas en compte le phénomène de nucléation.

(2) Le modèle développé, bien qu'il soit soumis à des hypothèses restrictives, décrit bien la réalité de l'évolution du rayon d'une bulle.

(3) Le modèle ne prend pas en compte le phénomène de diffusion des gaz à travers la matrice fondue. Il est à noter que ce phénomène est moins influent sur l'étape de l'expansion d'une bulle. Il devient, en revanche, prédominant lors des deuxième et troisième étapes de la courbe d'évolution. Dans la pratique, pour obtenir une morphologie cellulaire correcte de la mousse, le procédé de moussage devrait s'arrêter avant la régression des bulles. La prise en compte de ce phénomène est donc secondaire dans la modélisation. Il est, cependant, envisagé d'intégrer cet aspect complexe au modèle dans les prochains travaux de simulation de l'opération de moussage.

\section{Conclusion}

Un modèle d'expansion d'une bulle dans une cellule élémentaire a été utilisé pour simuler, à l'echelle microscopique, l'expansion d'une mousse polymère par un agent porogène chimique dans le contexte du rotomoulage. Dans ce procédé, l'expansion des bulles est provoquée par la décomposition de l'agent porogène qui représente l'élément moteur de la croissance de la phase gazeuse du matériau cellulaire.

Les observations microscopiques ont mis enévidencel'hétérogénéité des phénomènes de la nucléation et de l'expansion des bulles. La cause de cette hétérogénéité est attribuée au gradient du champ de température et à la distribution aléatoire des particules de l'agent porogène dans la matrice. Toutefois, il est à noter que la coalescence entre les bulles voisines a été très rarement observée.

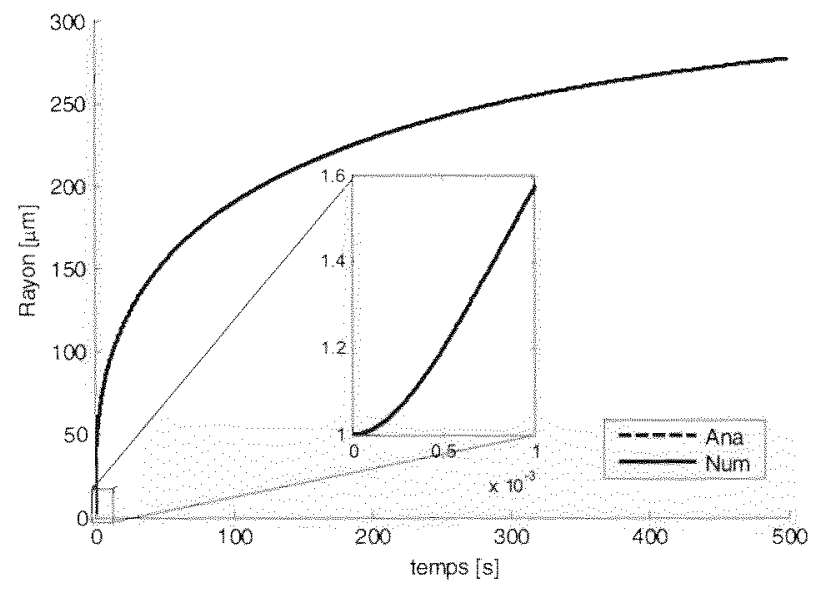

Fig. 14. Comparaison entre la solution analytique et la solution numérique du rayon $R(t)$ de la bulle.

Fig. 14. Comparison of analytical and numerical solutions of bubble radius $R(t)$.

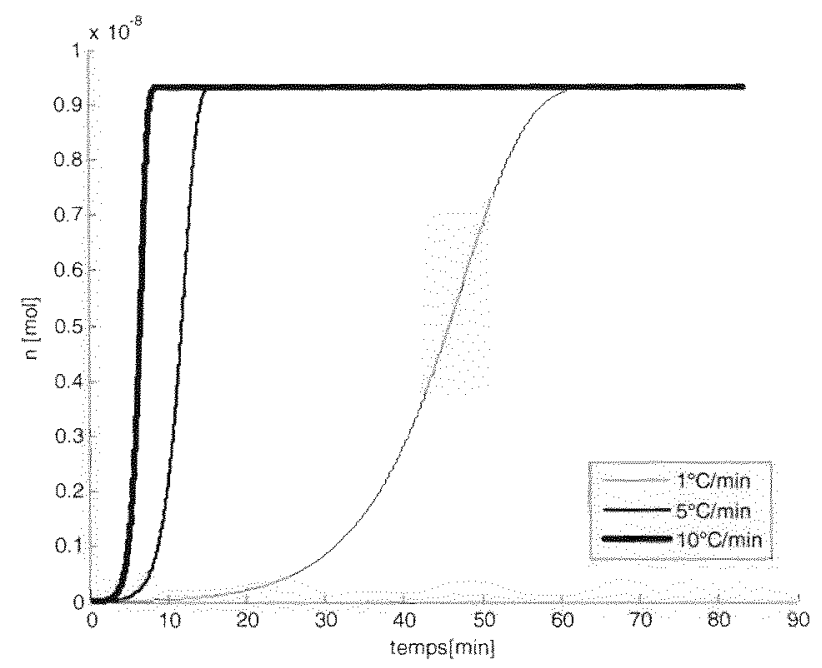

Fig. 15. Effet de la rampe de température sur nombre de moles de gaz $n(t)$.

Fig. 15. Temperature rate effect on the gas moles number $n(t)$.

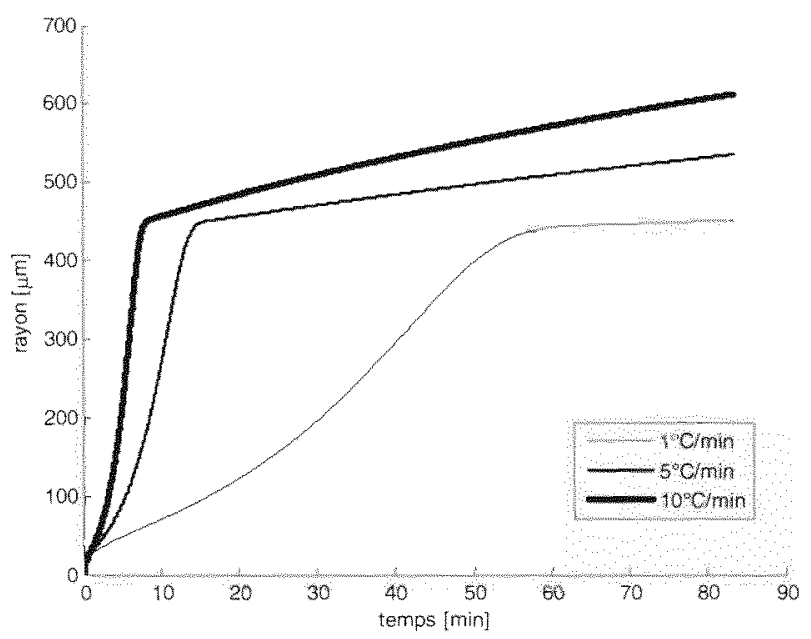

Fig. 16. Effet de la rampe de température sur le rayon $R(t)$ de la bulle.

Fig. 16. Temperature rate effect on the bubble radius $R(t)$. 


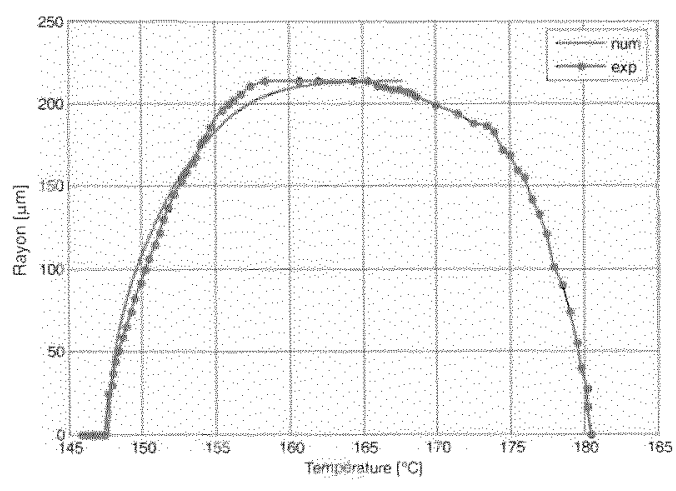

Fig. 17. Comparaison entre le modèle numérique et les mesures expérimentales du rayon de la bulle 1 de la figure 6 .

Fig. 17. Comparison between numertal model and experimental measurement of bubble 1 radius of figure 6.

La forme géométrique des bulles dépend de la présence des charges dans la matrice fondue et de leur position par rapport au bord de la cellule.

Un modèle de la cinétique chimique de la réaction de décomposition de l'agent porogène a été développé pour foumir l'évolution du nombre de moles de gaz engendrées en fonction de la température. Le modèle de l'expansion présenté rend compte de l'effet de la température sur le processus de décomposition et sur la vitesse $\mathrm{d}^{r}$ expansion des bulles et ce, conformément aux résultats expérimentaux. Bien qu'il soit soumis à des hypothèses restrictives, il présente une bonne corrélation avec les mesures en 2D de la croissance des rayons des bulles observées par microscope.

Les principales perspectives concernent la prise en compte des phénomènes physiques comme la diffusion et la dilatation thermique des gaz au sein de la bulle ainsi que l'intégration de la thermo-dépendance de la tension superficielle. Une approche éléments finis permettrait de procéder à la simulation d'un échantillon de mousse pour améliorer les performances du procédé.

\section{Références}

[1] B. Ben Lazreg, F. Chinesta, G. Doulin, Ph. Mazabraud, A. Sanchez, A. Tcharkhtchi, Mechanism of blowing agent decomposition during rotational foam molding, MoDest, 3rd Intemational Conference, 30/08/04 au 2/09/04, Lyon- France

[2] G. Liu, C.B. Park, J.A. Lefas, Polym. Eng. Sct. 38 (1998) 1997-2009

[3] R. Pop-lliev, G.M. Rizvi, C.B. Park, Polym. Eng. Sci. 43 (200) 40-54

[4] F. Chinesta, G. Chaidron, M. Godet, A. Bermudez, Evaluation d'un modèle numérique simplifié de l'expansion des mousses, Colloque annuel du groupe français de rhéologien ${ }^{\circ} 35$, Grenoble, France, Vol. 17. Issue 1, pp. 403-411, 23/10/2000

[5] M. Amon, C.D. Denson, Polym. Eng. Sci. 26 (1986) 255-267

[6] J. Bruchon, T. Coupez, Mécanique Es Industries 4 (2003) 331-338

[7] I. Bikard, J. Bruchon, T. Coupez, B. Vergnes, Mechanical behaviour of cellular solids: Numerical prediction of the foam structure of polymeric materials by direct $3 \mathrm{D}$ simulation of their expansion by chemical reaction based on a multidomain method, EUROMECH Colloquium 459, Nancy, France (07/06/2004), I. Mater. Sci. 40 (2005) $5875-5881$

[8] M.A. Shafi, K. Joshi, R.W. Flumerflet, Chem. Eng. Sci. 52 (1997) 635-644

[9] M.A. Shafi, J.G. Lee, R.W. Flumerfelt, Polym. Eng. Sci. 36 (2004) 1950-1959

[10] M.A. Shafi, R.W. Flumerfelt, Chem. Eng. Sci. $52(1997) 627-633$

[11] V. Talanquer, D.W. Oxtoby, J. Chem. Phys. 102 (1995) 2156-2164

[12] A. Arefmanesh, S.G. Advani, Polym. Eng. Sci. 35 (2004) 252-260 